\title{
Detection of NCOA2/3 Gene Fusions in Uterine Tumors Resembling Ovarian Sex Cord Tumors (UTROSCT)
}

Andre Pinto1, Ricardo R. Lastra², Lauren L. Ritterhouse ${ }^{3}$, Jeremy Segal ${ }^{2}$, Thomas Krausz ${ }^{2}$, Jennifer A. Bennett ${ }^{2}$

${ }^{1}$ University of Miami, Miami, FL, USA; ${ }^{2}$ University of Chicago, Chicago, IL, USA,

${ }^{3}$ Massachusetts General Hospital, Boston, MA, USA

\section{Background}

Uterine tumors resembling ovarian sex cord tumors (UTROSCT) are rare mesenchymal neoplasms of uncertain histogenesis that are challenging to diagnose due to their morphological and immunohistochemical overlap with more conventional entities. While DNA sequencing has failed to identify recurring mutations in these tumors, RNA sequencing recently detected NCOA2/3 fusions in two small series.

\section{Methods}

We retrospectively evaluated the clinicopathological and immunohistochemical features of two UTROSCTs, and performed RNA sequencing to detect gene fusions.

\section{Results}

The patients were 52 and 57 years old, tumors measured 5 and $12 \mathrm{~cm}$, and were confined to the myometrium. Both showed multiple histologic patterns including diffuse, cord-like and trabecular, with rhabdoid cells focally present. One UTROSCT had significant cytologic atypia and brisk mitotic activity. Variable immunohistochemical expression for sex cord and epithelial markers was noted. RNA sequencing detected an ESR1-NCOA3 fusion in one tumor, whereas the other had a GREB1-NCOA2 rearrangement. The former patient is alive and well 5 months after diagnosis, while the latter recurred two years later, and is currently alive with disease (54 months after original diagnosis). These findings are summarized in the table below.

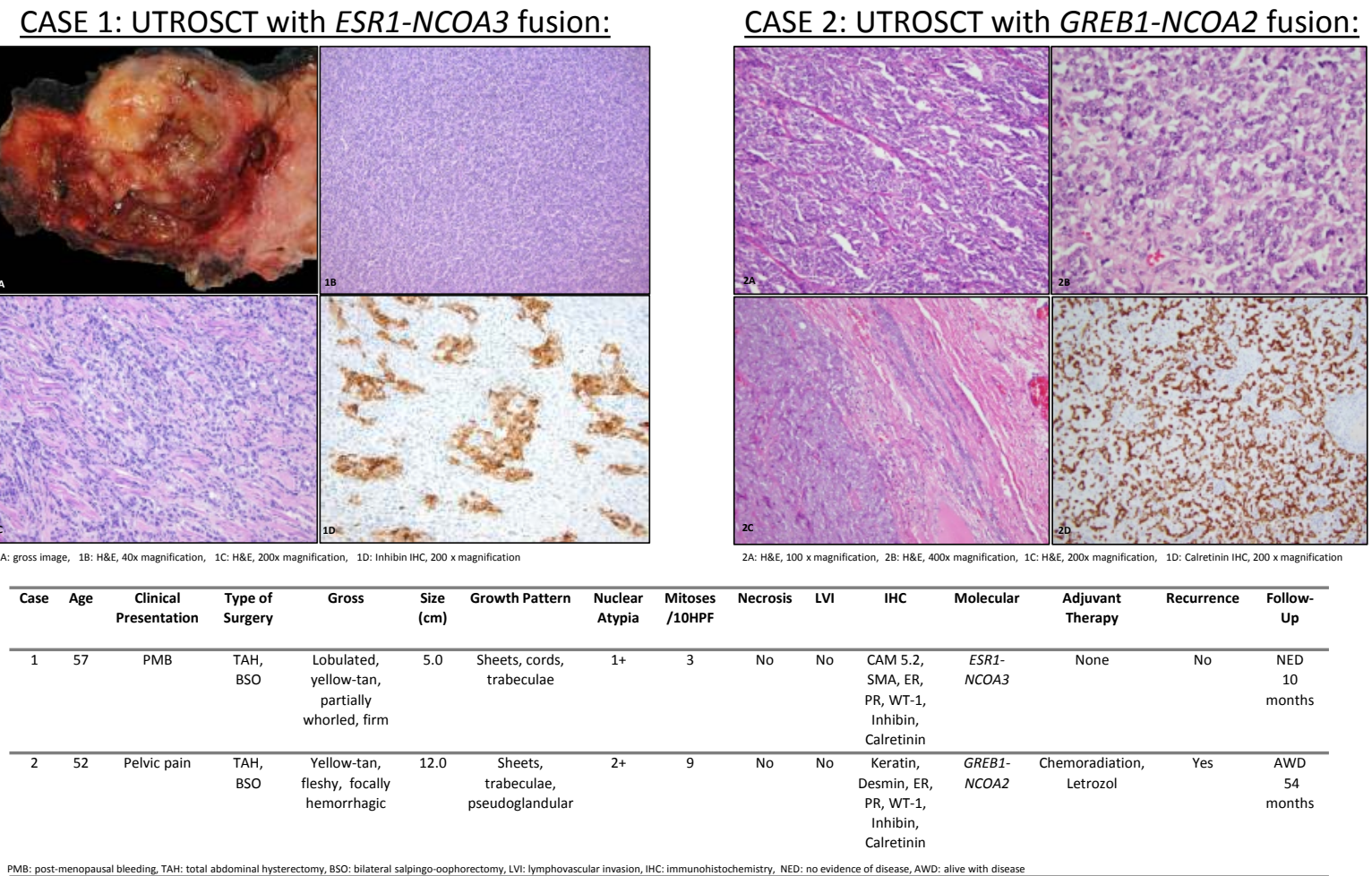

\section{Conclusion}

The detection of NCOA2/3 fusions in two additional UTROSCTs further supports this rearrangement as a characteristic finding in these rare tumors. Additional studies are warranted to determine its sensitivity and specificity in UTROSCTs compared to other gynecologic neoplasms. 